\title{
Semi-Automated Optimization of the CHARMM36 Lipid Force Field to Include Explicit Treatment of Long-Range Dispersion
}

\section{Supporting Information}

Yalun Yu, ${ }^{\mathrm{a}, \mathrm{b}}$ Andreas Krämer, ${ }^{\mathrm{b}}$ Richard M. Venable, ${ }^{\mathrm{b}}$ Andrew C. Simmonett, ${ }^{\mathrm{b}}$ Alexander D. MacKerell, Jr., ${ }^{c}$ Jeffery B. Klauda, ${ }^{*, a, d}$ Richard W. Pastor, ${ }^{*}$, and Bernard R. Brooks ${ }^{*}, \mathrm{~b}$ aBiophysics Graduate Program and ${ }^{\mathrm{d} D e p a r t m e n t}$ of Chemical and Biomolecular Engineering, University of Maryland, College Park, Maryland 20742, USA

baboratory of Computational Biology, National Heart, Lung and Blood Institute, National Institutes of Health, Bethesda, MD 20892, USA

cDepartment of Pharmaceutical Sciences, School of Pharmacy, University of Maryland, Baltimore, Maryland 21201, USA

Table S1. Parameters subject to change for the two optimizations. Atom notations shown in Figure $\mathrm{S} 1$. Asterisk sign stands for $\mathrm{A} / \mathrm{B} / \mathrm{C}$.

\begin{tabular}{|l|l|l|}
\hline Optimization Name & Global & Linkage \\
\hline Charges & $\mathrm{N}, \mathrm{H} 13^{*}, \mathrm{H} 14^{*}, \mathrm{H} 15^{*}, \mathrm{C} 13$, & $\mathrm{C} 2, \mathrm{HS}, \mathrm{O} 21, \mathrm{C} 21, \mathrm{O} 22, \mathrm{C} 22$, \\
& $\mathrm{C} 14, \mathrm{C} 15, \mathrm{C} 12, \mathrm{H} 12^{*}, \mathrm{C} 11$, & $\mathrm{H} 2 \mathrm{R}, \mathrm{H} 2 \mathrm{~S}, \mathrm{C} 3, \mathrm{HX}, \mathrm{HY}, \mathrm{O} 31$, \\
& $\mathrm{H} 11^{*}, \mathrm{P}, \mathrm{O} 13, \mathrm{O} 14, \mathrm{O} 11$, & $\mathrm{C} 31, \mathrm{O} 32, \mathrm{C} 32, \mathrm{H} 2 \mathrm{X}, \mathrm{H} 2 \mathrm{Y}$ \\
& $\mathrm{O} 12, \mathrm{C} 1, \mathrm{HA}, \mathrm{HB}, \mathrm{C} 2, \mathrm{HS}$, & \\
& $\mathrm{O} 21, \mathrm{C} 21, \mathrm{O} 22, \mathrm{C} 22, \mathrm{H} 2 \mathrm{R}$, & \\
& $\mathrm{H} 2 \mathrm{~S}, \mathrm{C} 3, \mathrm{HX}, \mathrm{HY}, \mathrm{O} 31$, & \\
& $\mathrm{C} 31, \mathrm{O} 32, \mathrm{C} 32, \mathrm{H} 2 \mathrm{X}, \mathrm{H} 2 \mathrm{Y}$ & \\
\hline$\varepsilon$ & $\mathrm{N}, \mathrm{H} 13^{*}, \mathrm{H} 14^{*}, \mathrm{H} 15^{*}, \mathrm{C} 13$, & $\mathrm{O} 21, \mathrm{O} 22, \mathrm{O} 31, \mathrm{C} 32$ \\
& $\mathrm{C} 14, \mathrm{C} 15, \mathrm{H} 12^{*}, \mathrm{C} 11, \mathrm{H} 11^{*}$, & \\
& $\mathrm{P}, \mathrm{O} 11, \mathrm{O} 12, \mathrm{O} 21, \mathrm{C} 21, \mathrm{O} 22$, & \\
& $\mathrm{O} 31, \mathrm{C} 31, \mathrm{C} 32$ \\
\hline$R_{\min } / 2$ & $\mathrm{~N}, \mathrm{H} 13^{*}, \mathrm{H} 14^{*}, \mathrm{H} 15^{*}, \mathrm{C} 13$, & $\mathrm{O} 21, \mathrm{O} 22, \mathrm{O} 31, \mathrm{C} 32$ \\
& $\mathrm{C} 14, \mathrm{C} 15, \mathrm{H} 12^{*}, \mathrm{C} 11, \mathrm{H} 11^{*}$, & \\
& $\mathrm{P}, \mathrm{O} 11, \mathrm{O} 12, \mathrm{O} 21, \mathrm{C} 21, \mathrm{O} 22$, & \\
& $\mathrm{O} 31, \mathrm{C} 31, \mathrm{C} 32$ & \\
\hline
\end{tabular}


Table S2-a. Rules for charge redistribution for the Global optimization. When the charge on the center atom(s) is changed, the opposite change is evenly distributed to the atom(s) to exchange charge with. The transfer of charge is always within an integer charge group defined in CHARMM. Equivalent atomic sites from group 3 and group 4 are listed in the same rows.

\begin{tabular}{|c|c|}
\hline center atom(s) & atom(s) to exchange charge with \\
\hline \multicolumn{2}{|l|}{ group 1} \\
\hline $\mathrm{N}$ & $\mathrm{C} 13, \mathrm{C} 14, \mathrm{C} 15, \mathrm{C} 12$ \\
\hline H13*, H14*, H15* & $\mathrm{H} 12 *$ \\
\hline $\mathrm{C} 13, \mathrm{C} 14, \mathrm{C} 15$ & H13*, H14*, H15* \\
\hline $\mathrm{C} 12$ & $\mathrm{H} 12^{*}$ \\
\hline \multicolumn{2}{|l|}{ group 2} \\
\hline $\mathrm{C} 11$ & $\mathrm{P}$ \\
\hline H11* & C11 \\
\hline $\mathrm{P}$ & $\mathrm{O} 13, \mathrm{O} 14, \mathrm{O} 11, \mathrm{O} 12$ \\
\hline $\mathrm{O} 13, \mathrm{O} 14$ & $\mathrm{O} 11, \mathrm{O} 12$ \\
\hline $\mathrm{O} 11, \mathrm{O} 12$ & $\mathrm{C} 1$ \\
\hline C1 & $\mathrm{HA}, \mathrm{HB}$ \\
\hline \multicolumn{2}{|l|}{ group 3 and group 4} \\
\hline $\mathrm{C} 2$ & HS \\
\hline $\mathrm{O} 21 / \mathrm{O} 31$ & $\mathrm{C} 2 / \mathrm{C} 3$ \\
\hline $\mathrm{C} 21 / \mathrm{C} 22$ & $(\mathrm{O} 21, \mathrm{O} 22, \mathrm{C} 22) /(\mathrm{O} 31, \mathrm{O} 32, \mathrm{C} 32)$ \\
\hline $\mathrm{O} 22$ / O32 & $\mathrm{C} 21 / \mathrm{C} 31$ \\
\hline $\mathrm{C} 22$ / C32 & $\mathrm{C} 21$ / C31 \\
\hline$(\mathrm{H} 2 \mathrm{R}, \mathrm{H} 2 \mathrm{~S}) /(\mathrm{H} 2 \mathrm{X}, \mathrm{H} 2 \mathrm{Y})$ & $\mathrm{C} 22$ / C32 \\
\hline $\mathrm{C} 3$ & HX, HY \\
\hline
\end{tabular}

Table S2-b. Rules for charge redistribution for the Linkage optimization. The way of exchanging charge is described in the caption of Table S2-a. Equivalent atomic sites from group 3 and group 4 are listed in the same rows.

\begin{tabular}{|c|c|}
\hline center atom(s) & atom(s) to exchange charge with \\
\hline \multicolumn{2}{|l|}{ group 3 and group 4} \\
\hline $\mathrm{C} 2 / \mathrm{C} 3$ & $\mathrm{O} 21 / \mathrm{O} 31$ \\
\hline HS & $\mathrm{C} 2$ \\
\hline $\mathrm{O} 21 / \mathrm{O} 31$ & $(\mathrm{C} 2, \mathrm{C} 21) /(\mathrm{C} 3, \mathrm{C} 31)$ \\
\hline $\mathrm{C} 21 / \mathrm{C} 22$ & $(\mathrm{O} 21, \mathrm{O} 22, \mathrm{C} 22) /(\mathrm{O} 31, \mathrm{O} 32, \mathrm{C} 32)$ \\
\hline $\mathrm{O} 22 / \mathrm{O} 32$ & $(\mathrm{O} 21, \mathrm{C} 21, \mathrm{C} 22) /(\mathrm{O} 31, \mathrm{C} 31, \mathrm{C} 32)$ \\
\hline $\mathrm{C} 22$ / C32 & $\mathrm{C} 21 / \mathrm{C} 31$ \\
\hline$(\mathrm{H} 2 \mathrm{R}, \mathrm{H} 2 \mathrm{~S}) /(\mathrm{H} 2 \mathrm{X}, \mathrm{H} 2 \mathrm{Y})$ & $\mathrm{C} 22$ / C32 \\
\hline
\end{tabular}


Table S3. Default weight factors for different parameter types in each optimization cycle.

\begin{tabular}{|l|l|l|l|}
\hline optimization cycle & $q$ & $\varepsilon$ & $R_{\min } / 2$ \\
\hline Global-1 & 4/e & 6/percentage & 6/percentage \\
\hline Global-2 & 4/e & 6/percentage & 6/percentage \\
\hline Global-3 & $16 / \mathrm{e}$ & 24/percentage & 24/percentage \\
\hline Linkage-1 & 4/e & 6/percentage & 6/percentage \\
\hline Linkage-2 & 4/e & 6/percentage & 6/percentage \\
\hline
\end{tabular}

Table S4. $S_{\mathrm{b}}$ in eq 5 for each optimization cycle.

\begin{tabular}{|l|l|}
\hline optimization cycle & $S_{\mathrm{b}}$ \\
\hline Global-1 & 500 \\
\hline Global-2 & 500 \\
\hline Global-3 & 2000 \\
\hline Linkage-1 & 500 \\
\hline Linkage-2 & 500 \\
\hline
\end{tabular}


Table S5. Partial atomic charges (e) for each optimization cycle. "G" stands for Global and "L" stands for Linkage.

\begin{tabular}{|c|c|c|c|c|c|c|c|}
\hline atom name & atom type & original & G-1 & G-2 & G-3 & L-1 & L-2 \\
\hline $\mathrm{N}$ & NTL & -0.60 & -0.5959 & -0.6030 & -0.6030 & -0.6 & -0.6 \\
\hline $\mathrm{C} 13$ & CTL5 & -0.35 & -0.3468 & -0.3451 & -0.3451 & -0.35 & -0.35 \\
\hline H13A & HL & 0.25 & 0.2506 & 0.2542 & 0.2542 & 0.25 & 0.25 \\
\hline H13B & HL & 0.25 & 0.2506 & 0.2542 & 0.2542 & 0.25 & 0.25 \\
\hline H13C & HL & 0.25 & 0.2506 & 0.2542 & 0.2542 & 0.25 & 0.25 \\
\hline $\mathrm{C} 14$ & CTL5 & -0.35 & -0.3468 & -0.3451 & -0.3451 & -0.35 & -0.35 \\
\hline H14A & HL & 0.25 & 0.2506 & 0.2542 & 0.2542 & 0.25 & 0.25 \\
\hline H14B & HL & 0.25 & 0.2506 & 0.2542 & 0.2542 & 0.25 & 0.25 \\
\hline $\mathrm{H} 14 \mathrm{C}$ & HL & 0.25 & 0.2506 & 0.2542 & 0.2542 & 0.25 & 0.25 \\
\hline C15 & CTL5 & -0.35 & -0.3468 & -0.3451 & -0.3451 & -0.35 & -0.35 \\
\hline H15A & HL & 0.25 & 0.2506 & 0.2542 & 0.2542 & 0.25 & 0.25 \\
\hline H15B & HL & 0.25 & 0.2506 & 0.2542 & 0.2542 & 0.25 & 0.25 \\
\hline $\mathrm{H} 15 \mathrm{C}$ & HL & 0.25 & 0.2506 & 0.2542 & 0.2542 & 0.25 & 0.25 \\
\hline $\mathrm{C} 12$ & CTL2 & -0.10 & -0.0680 & -0.0551 & -0.0551 & -0.1 & -0.1 \\
\hline H12A & HL & 0.25 & 0.2245 & 0.2027 & 0.2027 & 0.25 & 0.25 \\
\hline H12B & HL & 0.25 & 0.2245 & 0.2027 & 0.2027 & 0.25 & 0.25 \\
\hline $\mathrm{C} 11$ & CTL2 & -0.08 & -0.0914 & -0.0726 & -0.0793 & -0.08 & -0.08 \\
\hline H11A & HAL2 & 0.09 & 0.0869 & 0.0687 & 0.0664 & 0.09 & 0.09 \\
\hline H11B & HAL2 & 0.09 & 0.0869 & 0.0687 & 0.0664 & 0.09 & 0.09 \\
\hline $\mathrm{P}$ & PL & 1.50 & 1.5149 & 1.5229 & 1.5308 & 1.5 & 1.5 \\
\hline $\mathrm{O} 13$ & $\mathrm{O} 2 \mathrm{~L}$ & -0.78 & -0.7898 & -0.7874 & -0.7816 & -0.78 & -0.78 \\
\hline $\mathrm{O} 14$ & $\mathrm{O} 2 \mathrm{~L}$ & -0.78 & -0.7898 & -0.7874 & -0.7816 & -0.78 & -0.78 \\
\hline O11 & OSLP & -0.57 & -0.5423 & -0.5295 & -0.5307 & -0.57 & -0.57 \\
\hline $\mathrm{O} 12$ & OSLP & -0.57 & -0.5423 & -0.5295 & -0.5307 & -0.57 & -0.57 \\
\hline $\mathrm{C} 1$ & CTL2 & -0.08 & -0.1001 & -0.1484 & -0.1481 & -0.08 & -0.08 \\
\hline HA & HAL2 & 0.09 & 0.0834 & 0.0972 & 0.0942 & 0.09 & 0.09 \\
\hline $\mathrm{HB}$ & HAL2 & 0.09 & 0.0834 & 0.0972 & 0.0942 & 0.09 & 0.09 \\
\hline $\mathrm{C} 2$ & CTL1 & 0.17 & 0.1412 & 0.1020 & 0.0988 & 0.1638 & 0.1339 \\
\hline HS & HAL1 & 0.09 & 0.1188 & 0.1580 & 0.1626 & 0.1092 & 0.1023 \\
\hline $\mathrm{O} 21$ & OSL & -0.49 & -0.4904 & -0.4904 & -0.4919 & -0.4992 & -0.4739 \\
\hline C21 & CL & 0.90 & 0.8793 & 0.8604 & 0.8579 & 0.8669 & 0.8445 \\
\hline $\mathrm{O} 22$ & OBL & -0.63 & -0.6507 & -0.6607 & -0.6560 & -0.6391 & -0.6272 \\
\hline $\mathrm{C} 22$ & CTL2 & -0.22 & -0.1770 & -0.1555 & -0.1565 & -0.1873 & -0.1652 \\
\hline $\mathrm{H} 2 \mathrm{R}$ & HAL2 & 0.09 & 0.0894 & 0.0931 & 0.0926 & 0.0928 & 0.0928 \\
\hline $\mathrm{H} 2 \mathrm{~S}$ & HAL2 & 0.09 & 0.0894 & 0.0931 & 0.0926 & 0.0928 & 0.0928 \\
\hline C3 & CTL2 & 0.08 & 0.0830 & 0.1125 & 0.1139 & 0.1026 & 0.0302 \\
\hline HX & HAL2 & 0.09 & 0.0885 & 0.0738 & 0.0738 & 0.0852 & 0.1030 \\
\hline
\end{tabular}




\begin{tabular}{|c|c|c|c|c|c|c|c|}
\hline HY & HAL2 & 0.09 & 0.0885 & 0.0738 & 0.0738 & 0.0852 & 0.1030 \\
O31 & OSL & -0.49 & -0.4904 & -0.4904 & -0.4919 & -0.4992 & -0.4739 \\
C31 & CL & 0.90 & 0.8793 & 0.8604 & 0.8579 & 0.8669 & 0.8445 \\
O32 & OBL & -0.63 & -0.6507 & -0.6607 & -0.6560 & -0.6391 & -0.6272 \\
C32 & CTL2 & -0.22 & -0.1770 & -0.1555 & -0.1565 & -0.1873 & -0.1652 \\
H2X & HAL2 & 0.09 & 0.0894 & 0.0931 & 0.0926 & 0.0928 & 0.0928 \\
H2Y & HAL2 & 0.09 & 0.0894 & 0.0931 & 0.0926 & 0.0928 & 0.0928 \\
\hline
\end{tabular}


Table S6. $\varepsilon(\mathrm{kcal} / \mathrm{mol})$ for each optimization cycle. "G" stands for Global and "L" stands for Linkage. For the Linkage optimization, changed parameters are emphasized in bold.

\begin{tabular}{|c|c|c|c|c|c|c|c|}
\hline atom name & atom type & original & G-1 & G-2 & G-3 & L-1 & L-2 \\
\hline $\mathrm{N}$ & NTL & -0.2000 & -0.2000 & -0.2000 & -0.2000 & -0.2000 & -0.2000 \\
\hline C13 & CTL5 & -0.0800 & -0.0800 & -0.0800 & -0.0800 & -0.0800 & -0.0800 \\
\hline H13A & $\mathrm{HL}$ & -0.0460 & -0.0457 & -0.0455 & -0.0455 & -0.0460 & -0.0460 \\
\hline H13B & $\mathrm{HL}$ & -0.0460 & -0.0457 & -0.0455 & -0.0455 & -0.0460 & -0.0460 \\
\hline $\mathrm{H} 13 \mathrm{C}$ & $\mathrm{HL}$ & -0.0460 & -0.0457 & -0.0455 & -0.0455 & -0.0460 & -0.0460 \\
\hline C14 & CTL5 & -0.0800 & -0.0800 & -0.0800 & -0.0800 & -0.0800 & -0.0800 \\
\hline H14A & HL & -0.0460 & -0.0457 & -0.0455 & -0.0455 & -0.0460 & -0.0460 \\
\hline H14B & $\mathrm{HL}$ & -0.0460 & -0.0457 & -0.0455 & -0.0455 & -0.0460 & -0.0460 \\
\hline $\mathrm{H} 14 \mathrm{C}$ & $\mathrm{HL}$ & -0.0460 & -0.0457 & -0.0455 & -0.0455 & -0.0460 & -0.0460 \\
\hline $\mathrm{C} 15$ & CTL5 & -0.0800 & -0.0800 & -0.0800 & -0.0800 & -0.0800 & -0.0800 \\
\hline H15A & $\mathrm{HL}$ & -0.0460 & -0.0457 & -0.0455 & -0.0455 & -0.0460 & -0.0460 \\
\hline H15B & $\mathrm{HL}$ & -0.0460 & -0.0457 & -0.0455 & -0.0455 & -0.0460 & -0.0460 \\
\hline $\mathrm{H} 15 \mathrm{C}$ & HL & -0.0460 & -0.0457 & -0.0455 & -0.0455 & -0.0460 & -0.0460 \\
\hline $\mathrm{C} 12$ & CTL2 & -0.0560 & -0.0560 & -0.0560 & -0.0560 & -0.0560 & -0.0560 \\
\hline H12A & HL & -0.0460 & -0.0457 & -0.0455 & -0.0455 & -0.0460 & -0.0460 \\
\hline H12B & HL & -0.0460 & -0.0457 & -0.0455 & -0.0455 & -0.0460 & -0.0460 \\
\hline C11 & CTL2 & -0.0560 & -0.0560 & -0.0560 & -0.0560 & -0.0560 & -0.0560 \\
\hline H11A & HAL2 & -0.0280 & -0.0280 & -0.0280 & -0.0280 & -0.0280 & -0.0280 \\
\hline H11B & HAL2 & -0.0280 & -0.0280 & -0.0280 & -0.0280 & -0.0280 & -0.0280 \\
\hline $\mathrm{P}$ & PL & -0.5850 & -0.5850 & -0.5850 & -0.5850 & -0.5850 & -0.5850 \\
\hline $\mathrm{O} 13$ & $\mathrm{O} 2 \mathrm{~L}$ & -0.1200 & -0.1196 & -0.1194 & -0.1194 & -0.1200 & -0.1200 \\
\hline O14 & $\mathrm{O} 2 \mathrm{~L}$ & -0.1200 & -0.1196 & -0.1194 & -0.1194 & -0.1200 & -0.1200 \\
\hline O11 & OSLP & -0.1000 & -0.0996 & -0.0996 & -0.0996 & -0.1000 & -0.1000 \\
\hline $\mathrm{O} 12$ & OSLP & -0.1000 & -0.0996 & -0.0996 & -0.0996 & -0.1000 & -0.1000 \\
\hline $\mathrm{C} 1$ & CTL2 & -0.0560 & -0.0560 & -0.0560 & -0.0560 & -0.0560 & -0.0560 \\
\hline HA & HAL2 & -0.0280 & -0.0280 & -0.0280 & -0.0280 & -0.0280 & -0.0280 \\
\hline $\mathrm{HB}$ & HAL2 & -0.0280 & -0.0280 & -0.0280 & -0.0280 & -0.0280 & -0.0280 \\
\hline $\mathrm{C} 2$ & CTL1 & -0.0200 & -0.0200 & -0.0200 & -0.0200 & -0.0200 & -0.0200 \\
\hline HS & HAL1 & -0.0220 & -0.0220 & -0.0220 & -0.0220 & -0.0220 & -0.0220 \\
\hline $\mathrm{O} 21$ & OSL & -0.1000 & -0.0995 & -0.0991 & -0.0991 & -0.1000 & -0.1000 \\
\hline $\mathrm{C} 21$ & CL & -0.0700 & -0.0696 & -0.0693 & -0.0693 & -0.0700 & -0.0700 \\
\hline $\mathrm{O} 22$ & OBL & -0.1200 & -0.1189 & -0.1185 & -0.1185 & -0.1192 & -0.1192 \\
\hline $\mathrm{C} 22$ & CTL2 & -0.0560 & -0.0560 & -0.0560 & -0.0560 & -0.0560 & -0.0560 \\
\hline $\mathrm{H} 2 \mathrm{R}$ & HAL2 & -0.0280 & -0.0280 & -0.0280 & -0.0280 & -0.0280 & -0.0280 \\
\hline $\mathrm{H} 2 \mathrm{~S}$ & HAL2 & -0.0280 & -0.0280 & -0.0280 & -0.0280 & -0.0280 & -0.0280 \\
\hline $\mathrm{C} 3$ & CTL2 & -0.0560 & -0.0560 & -0.0560 & -0.0560 & -0.0560 & -0.0560 \\
\hline HX & HAL2 & -0.0280 & -0.0280 & -0.0280 & -0.0280 & -0.0280 & -0.0280 \\
\hline
\end{tabular}




\begin{tabular}{|l|c|c|c|c|c|c|c|}
\hline HY & HAL2 & -0.0280 & -0.0280 & -0.0280 & -0.0280 & -0.0280 & -0.0280 \\
O31 & OSL & -0.1000 & -0.0995 & -0.0991 & -0.0991 & -0.1000 & -0.1000 \\
C31 & CL & -0.0700 & -0.0696 & -0.0693 & -0.0693 & -0.0700 & -0.0700 \\
O32 & OBL & -0.1200 & -0.1189 & -0.1185 & -0.1185 & $\mathbf{- 0 . 1 1 9 2}$ & $\mathbf{- 0 . 1 1 9 2}$ \\
C32 & CTL2 & -0.0560 & -0.0560 & -0.0560 & -0.0560 & -0.0560 & -0.0560 \\
H2X & HAL2 & -0.0280 & -0.0280 & -0.0280 & -0.0280 & -0.0280 & -0.0280 \\
H2Y & HAL2 & -0.0280 & -0.0280 & -0.0280 & -0.0280 & -0.0280 & -0.0280 \\
\hline
\end{tabular}


Table S7. $R_{\min } / 2(\AA)$ for each optimization cycle. "G" stands for Global and "L" stands for Linkage. For the Linkage optimization, changed parameters are emphasized in bold.

\begin{tabular}{|c|c|c|c|c|c|c|c|}
\hline atom name & atom type & original & G-1 & G-2 & G-3 & L-1 & L-2 \\
\hline $\mathrm{N}$ & NTL & 1.850 & 1.8500 & 1.8500 & 1.8500 & 1.8500 & 1.8500 \\
\hline $\mathrm{C} 13$ & CTL5 & 2.060 & 2.0600 & 2.0600 & 2.0600 & 2.0600 & 2.0600 \\
\hline H13A & HL & 0.700 & 0.6951 & 0.6951 & 0.6951 & 0.7000 & 0.7000 \\
\hline H13B & $\mathrm{HL}$ & 0.700 & 0.6951 & 0.6951 & 0.6951 & 0.7000 & 0.7000 \\
\hline $\mathrm{H} 13 \mathrm{C}$ & $\mathrm{HL}$ & 0.700 & 0.6951 & 0.6951 & 0.6951 & 0.7000 & 0.7000 \\
\hline $\mathrm{C} 14$ & CTL5 & 2.060 & 2.0600 & 2.0600 & 2.0600 & 2.0600 & 2.0600 \\
\hline H14A & HL & 0.700 & 0.6951 & 0.6951 & 0.6951 & 0.7000 & 0.7000 \\
\hline H14B & $\mathrm{HL}$ & 0.700 & 0.6951 & 0.6951 & 0.6951 & 0.7000 & 0.7000 \\
\hline $\mathrm{H} 14 \mathrm{C}$ & $\mathrm{HL}$ & 0.700 & 0.6951 & 0.6951 & 0.6951 & 0.7000 & 0.7000 \\
\hline $\mathrm{C} 15$ & CTL5 & 2.060 & 2.0600 & 2.0600 & 2.0600 & 2.0600 & 2.0600 \\
\hline H15A & HL & 0.700 & 0.6951 & 0.6951 & 0.6951 & 0.7000 & 0.7000 \\
\hline H15B & HL & 0.700 & 0.6951 & 0.6951 & 0.6951 & 0.7000 & 0.7000 \\
\hline $\mathrm{H} 15 \mathrm{C}$ & $\mathrm{HL}$ & 0.700 & 0.6951 & 0.6951 & 0.6951 & 0.7000 & 0.7000 \\
\hline $\mathrm{C} 12$ & CTL2 & 2.010 & 2.0100 & 2.0100 & 2.0100 & 2.0100 & 2.0100 \\
\hline H12A & HL & 0.700 & 0.6951 & 0.6951 & 0.6951 & 0.7000 & 0.7000 \\
\hline H12B & HL & 0.700 & 0.6951 & 0.6951 & 0.6951 & 0.7000 & 0.7000 \\
\hline C11 & CTL2 & 2.010 & 2.0100 & 2.0100 & 2.0100 & 2.0100 & 2.0100 \\
\hline H11A & HAL2 & 1.340 & 1.3400 & 1.3400 & 1.3400 & 1.3400 & 1.3400 \\
\hline H11B & HAL2 & 1.340 & 1.3400 & 1.3400 & 1.3400 & 1.3400 & 1.3400 \\
\hline $\mathrm{P}$ & PL & 2.150 & 2.1500 & 2.1500 & 2.1500 & 2.1500 & 2.1500 \\
\hline $\mathrm{O} 13$ & $\mathrm{O} 2 \mathrm{~L}$ & 1.700 & 1.7516 & 1.7914 & 1.7000 & 1.7000 & 1.7000 \\
\hline O14 & $\mathrm{O} 2 \mathrm{~L}$ & 1.700 & 1.7516 & 1.7914 & 1.7000 & 1.7000 & 1.7000 \\
\hline O11 & OSLP & 1.650 & 1.6500 & 1.7094 & 1.7139 & 1.6500 & 1.6500 \\
\hline $\mathrm{O} 12$ & OSLP & 1.650 & 1.6500 & 1.7094 & 1.7139 & 1.6500 & 1.6500 \\
\hline $\mathrm{C} 1$ & CTL2 & 2.010 & 2.0100 & 2.0100 & 2.0100 & 2.0100 & 2.0100 \\
\hline HA & HAL2 & 1.340 & 1.3400 & 1.3400 & 1.3400 & 1.3400 & 1.3400 \\
\hline HB & HAL2 & 1.340 & 1.3400 & 1.3400 & 1.3400 & 1.3400 & 1.3400 \\
\hline $\mathrm{C} 2$ & CTL1 & 2.275 & 2.2750 & 2.2750 & 2.2750 & 2.2750 & 2.2750 \\
\hline HS & HAL1 & 1.320 & 1.3200 & 1.3200 & 1.3200 & 1.3200 & 1.3200 \\
\hline $\mathrm{O} 21$ & OSL & 1.650 & 1.6434 & 1.6132 & 1.6380 & 1.6809 & 1.6809 \\
\hline $\mathrm{C} 21$ & CL & 2.000 & 1.9848 & 1.9804 & 1.9750 & 2.0000 & 2.0000 \\
\hline $\mathrm{O} 22$ & OBL & 1.700 & 1.6735 & 1.6686 & 1.6789 & 1.6621 & 1.6470 \\
\hline $\mathrm{C} 22$ & CTL2 & 2.010 & 2.0100 & 2.0100 & 2.0100 & 2.0100 & 2.0100 \\
\hline $\mathrm{H} 2 \mathrm{R}$ & HAL2 & 1.340 & 1.3400 & 1.3400 & 1.3400 & 1.3400 & 1.3400 \\
\hline $\mathrm{H} 2 \mathrm{~S}$ & HAL2 & 1.340 & 1.3400 & 1.3400 & 1.3400 & 1.3400 & 1.3400 \\
\hline $\mathrm{C} 3$ & CTL2 & 2.010 & 2.0100 & 2.0100 & 2.0100 & 2.0100 & 2.0100 \\
\hline HX & HAL2 & 1.340 & 1.3400 & 1.3400 & 1.3400 & 1.3400 & 1.3400 \\
\hline
\end{tabular}




\begin{tabular}{|l|c|l|l|l|l|l|l|}
\hline HY & HAL2 & 1.340 & 1.3400 & 1.3400 & 1.3400 & 1.3400 & 1.3400 \\
O31 & OSL & 1.650 & 1.6434 & 1.6132 & 1.6380 & $\mathbf{1 . 6 8 0 9}$ & $\mathbf{1 . 6 8 0 9}$ \\
C31 & CL & 2.000 & 1.9848 & 1.9804 & 1.9750 & 2.0000 & 2.0000 \\
O32 & OBL & 1.700 & 1.6735 & 1.6686 & 1.6789 & $\mathbf{1 . 6 6 2 1}$ & $\mathbf{1 . 6 4 7 0}$ \\
C32 & CTL2 & 2.010 & 2.0100 & 2.0100 & 2.0100 & 2.0100 & 2.0100 \\
H2X & HAL2 & 1.340 & 1.3400 & 1.3400 & 1.3400 & 1.3400 & 1.3400 \\
H2Y & HAL2 & 1.340 & 1.3400 & 1.3400 & 1.3400 & 1.3400 & 1.3400 \\
\hline
\end{tabular}

Table S8. Real-space cutoff $\left(R_{\text {cut }}\right)$ dependence of $A_{l}$ for DPPC bilayer at $323.15 \mathrm{~K}$. Standard errors shown in parentheses.

\begin{tabular}{|l|l|l|l|l|l|l|l|}
\hline$R_{\text {cut }}(\AA)$ & 16 & 14 & 12 & 10 & 9 & 8 & 6 \\
\hline$A_{l}\left(\AA^{2}\right)$ & $62.7(0.3)$ & $62.4(0.2)$ & $63.0(0.4)$ & $62.8(0.3)$ & $62.4(0.3)$ & $62.6(0.3)$ & $64.0(0.3)$ \\
\hline
\end{tabular}




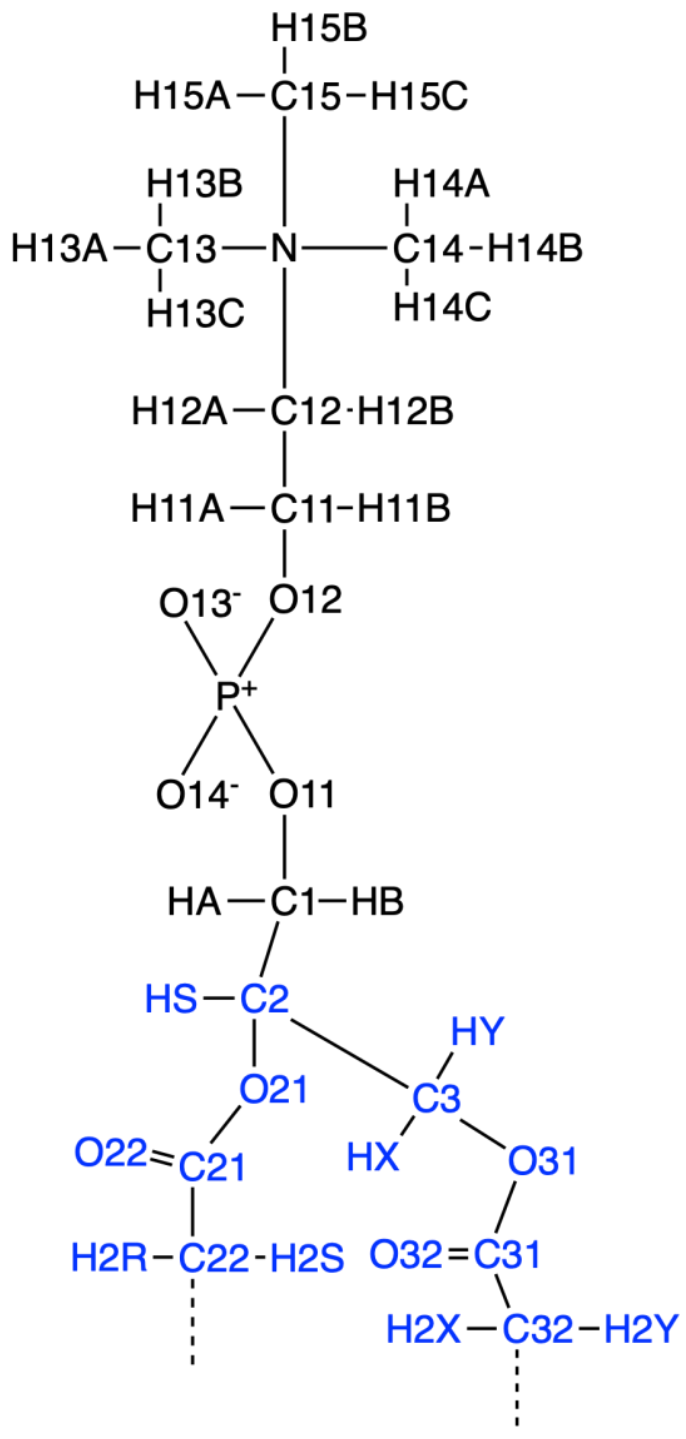

Figure S1. Atom naming of the head group for phosphatidylcholines. The blue part highlights the atoms parametrized in Linkage. 


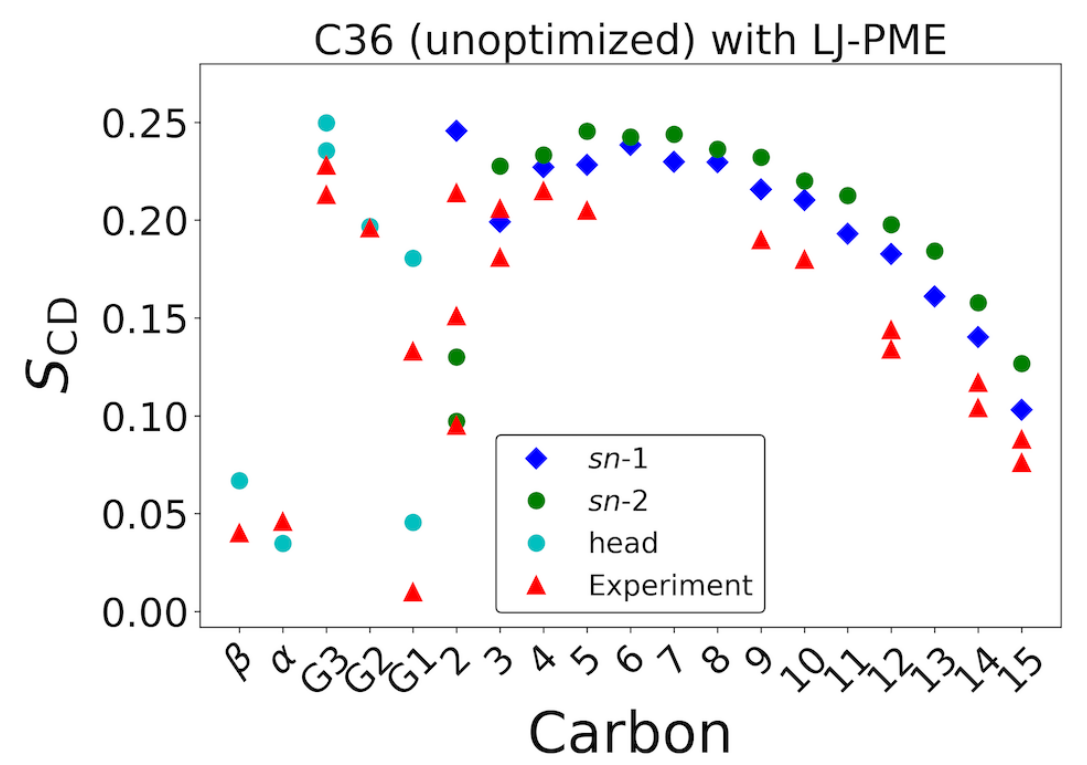

Figure S2. Deuterium order parameters of DPPC bilayer at 323.15 K (unoptimized). 
Initial
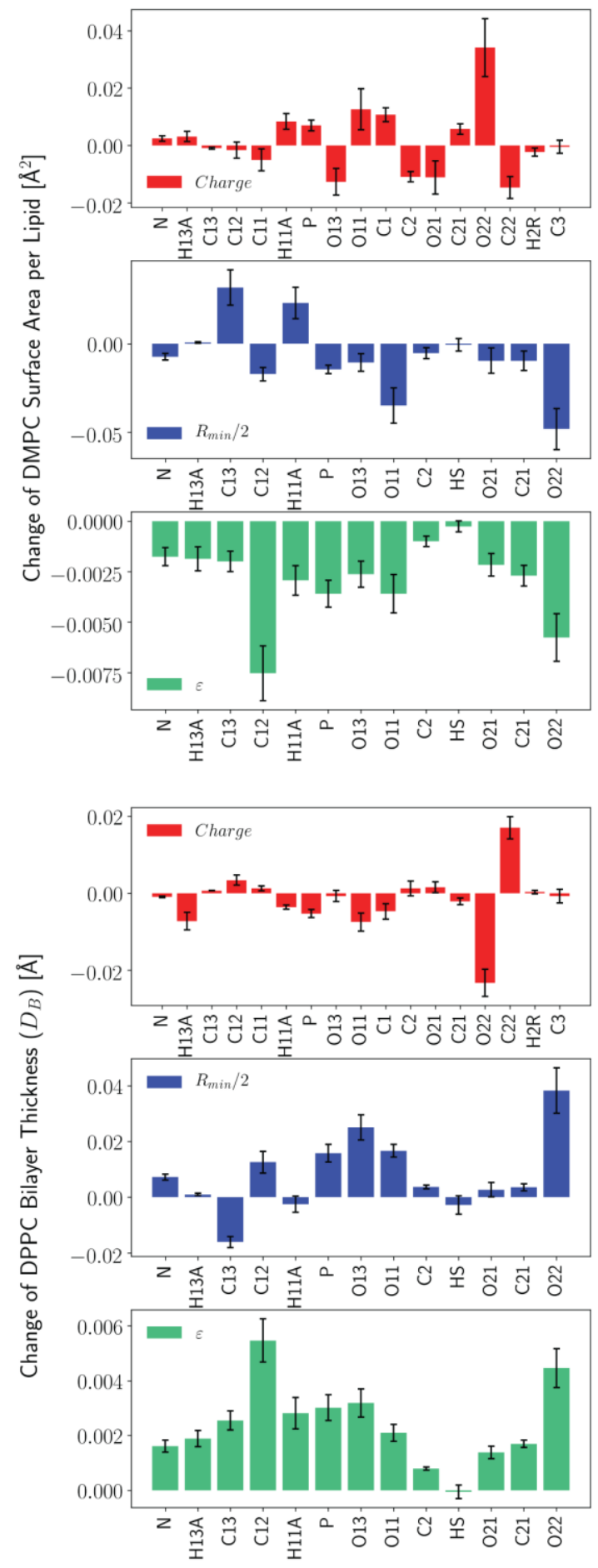

Final
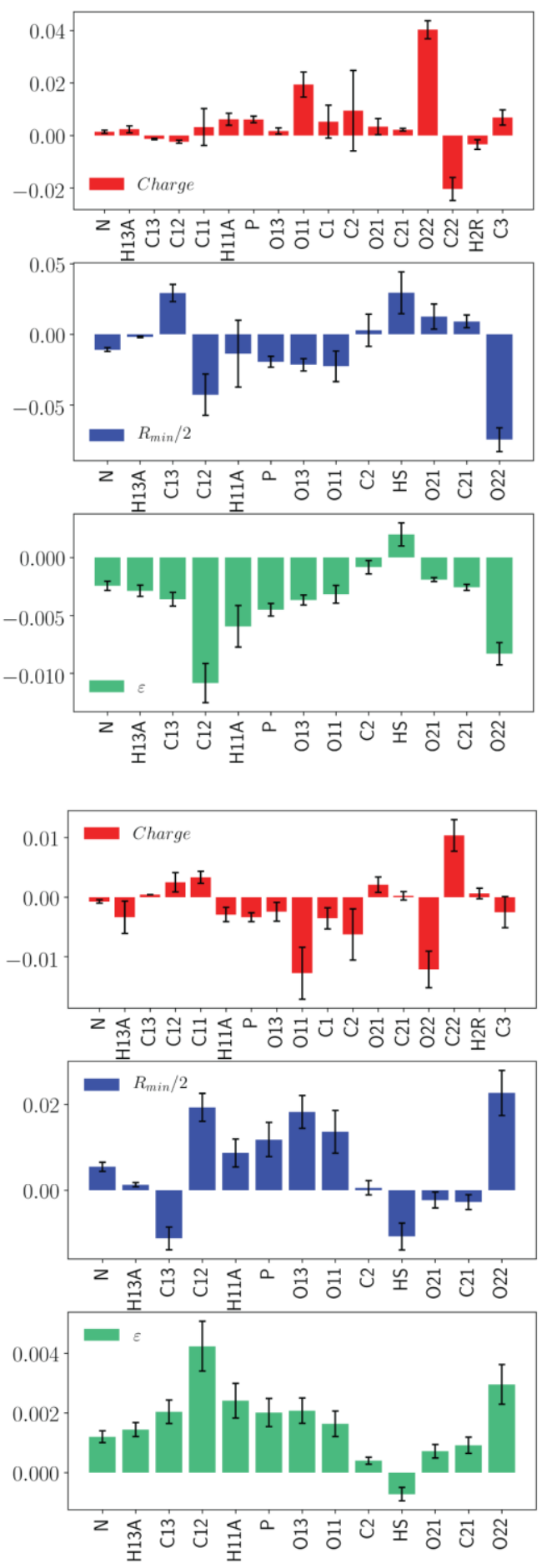
Initial
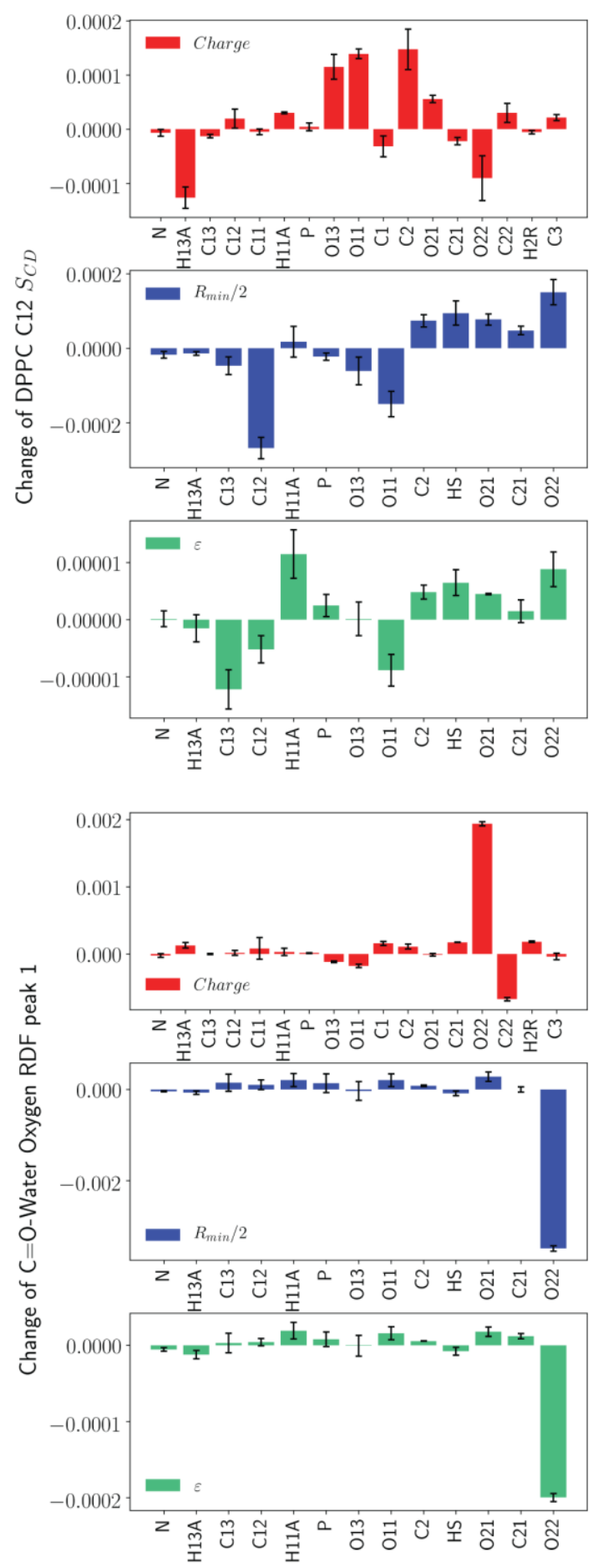

Final
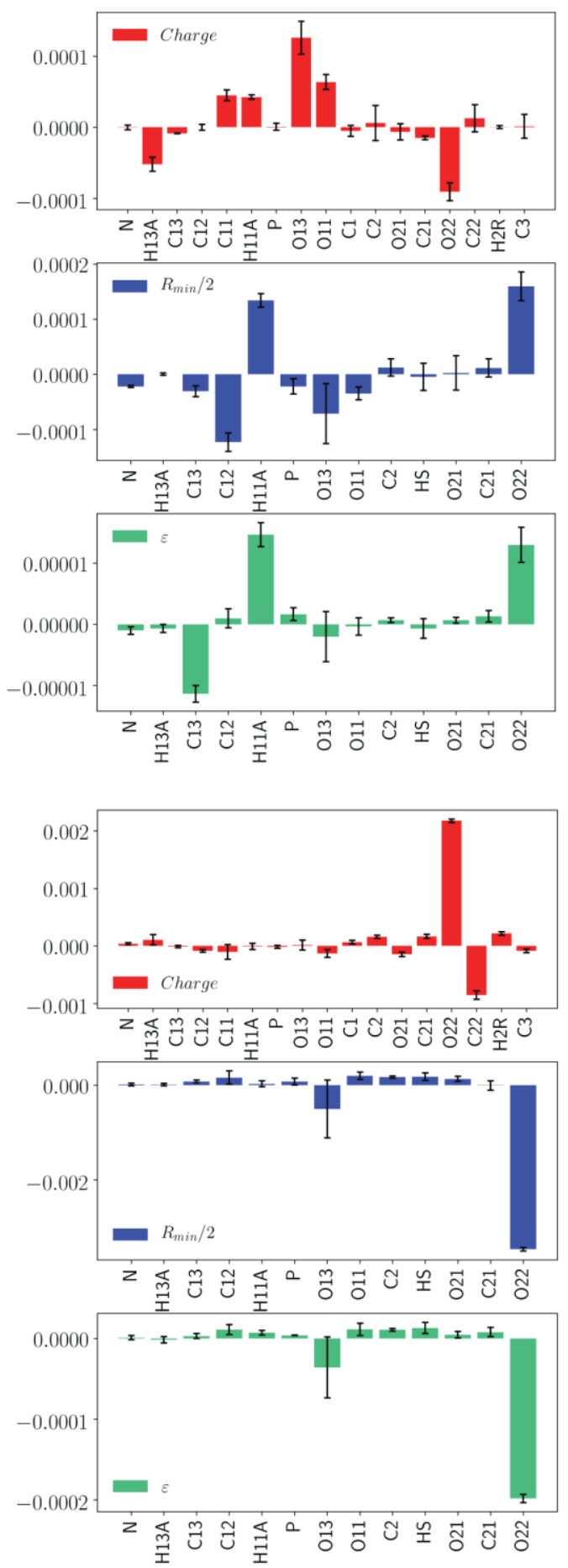

Figure S3. Parameter sensitivities for selected properties from the Global optimization. Standard deviations evaluated from trajectory blocks shown in black line with caps. The perturbation sizes used are 0.001 e (absolute value) for partial charge and $0.1 \%$ of the original parameter for $R_{\min } / 2$ and $\varepsilon$. 